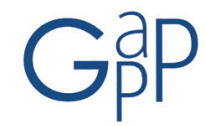

GESTIÓN Y ANÁLISIS DE POLÍTICAS PÚBLICAS, Nueva Época, nº 11 enero-junio 2014 ISSN: 1989-8991

DOI: http://dx.doi.org/10.24965/gapp.voi11.10177

\title{
Transformaciones y reformas en el sistema de pensiones argentino entre 2003 y 2013. Evaluando las políticas de inclusión más allá de lo contributivo
}

\author{
Giuseppe M. Messina \\ Instituto de Investigaciones Jurídicas y Sociales Gioja. Universidad de Buenos Aires \\ gm.messina@gmail.com
}

Recibido: 5 de febrero 2014 Aceptado: I 4 de marzo 2014

Resumen

En el caso de Argentina, los años posteriores a la crisis económica de 2001-2002 han estado marcados por el protagonismo creciente del Estado en la producción de bienestar. El objeto de este artículo es indagar sobre las políticas de inclusión implementadas por el gobierno argentino en el área de la protección social de las personas mayores de 65 años. El régimen previsional argentino ha sido tradicionalmente de tipo contributivo y de reparto. Las crisis económicas de las décadas anteriores y las reformas regresivas de los años go produjeron una notable reducción de la cobertura y de las prestaciones, en términos reales. En consecuencia, en los primeros años 2000 , una cuota significativa de los adultos mayores estaba excluida del sistema de protección social. Para hacer frente a esta situación, el gobierno desarrolló un conjunto de medidas de inclusión que favorecieron la ampliación de la cobertura del sistema previsional y una mejora del poder adquisitivo de las prestaciones. Sin embargo, se trató de soluciones transitorias que no han resuelto los problemas estructurales del sistema de protección social. En particular, la persistencia de un mercado laboral dual impide el correcto funcionamiento de las instituciones contributivas del Estado de bienestar argentino.

Palabras clave

Argentina, políticas sociales, pensiones, adultos mayores.

\section{Transformations and reforms in the Argentine pension system from 2003 through 2013. Assessing inclusion policies beyond the contributory principle}

\footnotetext{
Abstract

In Argentina, the years following the 2001-2002 economic crisis had been characterized by the growing importance of State intervention on welfare production. This paper looks into the inclusion policies implemented by the Argentine government in the area of social protection for people aged 65 and older. Traditionally, Argentine pension system had been contributory and had operated on a 'pay as you go' basis. Economic crises of earlier decades, and $90 s$ regressive reforms, caused a decline in coverage and real income transfers. Consequently, in the early 2000 s, a significant share of the older people was excluded from any pension system. In order to address this situation, the government developed a set of measures that favored inclusion extending the coverage of the pension system and improving the purchasing power of benefits. However, these were only temporal solutions since they have not solved the structural problems of the social protection system. In particular, the persistence of a dual labor market prevents the proper functioning of contributory institutions in the Argentine welfare State.
}

Keywords

Argentina, Social Policy, pensions, older persons. 


\section{INTRODUCCIÓN: ANTECEDENTES DE LAS REFORMAS DEL SISTEMA PREVISIONAL ARGENTINO BAJO EL KIRCHNERISMO}

Este trabajo aborda las transformaciones observadas en el sistema argentino de protección social para personas adultas mayores en la nueva etapa política que se abrió en ese país luego de la profunda crisis económica y social de 2001-2002. A partir de 2003, Argentina ha estado caracterizada por gobiernos estables del mismo signo político, en lo que se ha conocido como "kirchnerismo" en referencia a las presidencias de Néstor Kirchner (2003-2007) y Cristina Fernández (2008-2011; reelecta en 2011 hasta 2015). En el campo de las políticas sociales, los gobiernos kirchneristas han mostrado una fuerte atención a la situación de las personas excluidas de los tradicionales sistemas de protección social de tipo contributivo. En esta línea, junto a una renovación de los tradicionales programas asistenciales sobre la base de los modelos de transferencias condicionadas, que tanto éxito han tenido en otros países de la región', también se han implementado reformas que han favorecido una mayor flexibilidad en el acceso a las prestaciones contributivas.

Todos estos cambios se inscriben en un panorama de la región latinoamericana caracterizado por un "giro a la izquierda" en reacción a las consecuencias sociales de las políticas de inspiración neoliberal implementadas en los años 90. En el caso argentino, esa década estuvo marcada por un marcado empeoramiento de todos los indicadores sociales, en particular los laborales. La crisis del régimen de acumulación de tipo neoliberal a finales de 2001 significó una verdadera ruptura económica, social y política (cfr. Basualdo, 2011), durante la cual la tasa de pobreza alcanzó valores cercanos al $60 \%$ y el desempleo superó el $20 \% 2$. A partir de 2003, la recuperación económica y los efectos de las políticas de los gobiernos kirchneristas produjeron mejoras rápidas, tanto en los indicadores de ingresos (pobreza, desigualdad) como en los laborales. A finales de 2011, las tasas oficiales de pobreza y desempleo se habían ubicado por debajo del 10\%, mientras que la desigualdad de ingresos, medida por el índice de Gini, cayó cerca de diez puntos en porcentaje, recuperando los niveles de principios de los años $90^{3}$.

Naturalmente, el periodo de bonanza económica, entre otras cosas gracias al contexto internacional favorable, permitió que el gobierno argentino tuviera amplios márgenes de maniobra para implementar las reformas de la Seguridad Social que se describen en este trabajo. En paralelo, la recomposición del mercado laboral también consolidó los niveles de financiación de la Seguridad Social, gracias al incremento de las cotizaciones sociales. Con este contexto general de fondo, en este trabajo se discuten, en particular, las reformas que afectaron al componente de la Seguridad Social de protección contra los riesgos asociados a la vejez. En otras palabras, el foco del trabajo está en los mecanismos de seguro social que protegen de la pérdida de ingresos asociados al cese de la vida laboral activa, para las personas adultas mayores que hayan alcanzado la edad legal de jubilación (65 años para los varones y 60 años para las mujeres) $)^{4}$.

Como se irá mostrando a lo largo del artículo, en el caso argentino se produjo un incremento considerable del gasto social, que se reflejó en un fuerte incremento nominal de las pensiones erogadas, a la vez que se adoptaron medidas que extendieron la cobertura del sistema previsional a categorías previamente excluidas. Este objetivo fue perseguido con una estrategia institucional que reapropió para el Estado componentes de la seguridad social que habían sido cedidos al sector privado en la década anterior. Además, se relajaron las condiciones de acceso al sistema para las personas mayores excluidas por medio de una moratoria previsional; también se favoreció la afiliación de los trabajadores del sector no formal gracias al potenciamiento de la inspección laboral5 y una fuerte estrategia de simplificación y regularización tributaria para los trabajadores autónomos ${ }^{6}$ y del servicio doméstico; por último, se aumentó de manera considerable el monto de las pensiones erogadas, en particular las mínimas, permitiendo la recuperación de su poder adquisitivo.

Estos y otros cambios parecen marcar un verdadero cambio de paradigma respecto a las políticas neoliberales de los 9o, en un proceso que ha sido observado en numerosos países de la región (cfr. Rofman et al., 2014). El

1 Se trata de una nueva orientación de la política asistencial, a partir de los ejemplos paradigmáticos del "Bolsa Familia" en Brasil o el "Oportunidades" de México, que pretenden romper con la transmisión intergeneracional de la pobreza por medio del desarrollo del capital humano de la infancia. La marca característica de esta modalidad de política social es que las prestaciones están condicionadas a la inserción de los menores receptores en el sistema educativo y sanitario.

2 Los datos presentados en este párrafo provienen del Instituto Nacional de Estadística y Censos (INDEC).

3 El índice de Gini habría pasado de cifras superiores a 0,52 en 2002 a valores de poco superiores a 0,42 en 2012, en comparación a un valor de 0.45 en 1992. Fuente: SEDLAC (CEDLAS y Banco Mundial).

4 No se analizan en este trabajo otras políticas públicas que contribuyen, en igual medida, al bienestar de esta categoría, en particular todo lo relacionado al sistema de salud.

5 Puede mencionarse el Sistema Integral de Inspección del Trabajo y de la Seguridad Social de 2004.

6 Se hace aquí referencia al régimen tributario simplificado para pequeños contribuyentes, denominado monotributo, donde los pagos mensuales fijos, según la categoría, incluyen todos los aportes a la Seguridad Social. Tales aportes son aún más reducidos en el caso del "monotributo social", para trabajadores independientes (o en cooperativas de trabajo) en situación de vulnerabilidad social.

GESTIÓN Y ANÁLISIS DE POLÍTICAS PÚBLICAS, Nueva Época, nº 11 enero-junio 2014 ISSN: 1989-8991 - DOI: 10.24965/gapp.v0i11.10177 
caso argentino, no obstante, por su trayectoria histórica y por el contexto actual que experimenta el país, presenta numerosas peculiaridades que serán destacadas a lo largo del texto. En particular, no se examina sólo la mejora de la cobertura del sistema previsional argentino, es decir su extensión horizontal, resultado de los principios que regulan las condiciones de acceso al mismo (por ej. la cotización del trabajador o determinadas características sociodemográficas del individuo). También, se analizan los desafíos pendientes para garantizar su extensión vertical, es decir la adecuación del nivel de prestaciones que el actual régimen garantiza.

Más en general, la cuestión radica en si una determinada política social logra su objetivo de protección frente al riesgo social que justificó su implementación, es decir hasta qué punto el bienestar de los titulares es salvaguardado frente a las pérdidas ocasionadas en esa contingencia, en términos de menores ingresos y consumos. En general, en los sistemas de pensiones contributivos sólo los trabajadores del sector formal reciben protección al término de la vida laboral, quedando excluidos todos aquellos que no tienen ese status. En casos como el argentino, en los que los niveles de informalidad laboral son muy elevados, es necesario entonces adoptar estrategias de inclusión que cierren esa brecha, si se acepta como objetivo central el de garantizar un piso de protección universal para todas las personas al final de su vida laboral, en particular frente al riesgo de pobreza?.

Estas dimensiones de la política social expresan por lo tanto el grado con el que el Estado provee los bienes y servicios necesarios para cubrir las necesidades de un individuo o hogar (por ej. bajo forma de transferencias monetarias), frente a ciertas contingencias sociales (en este caso la vejez, el fin de la vida laboral activa), de forma independiente del estatus (por ej. laboral) del individuo. Se trata de lo que Esping-Andersen (1993) definió el efecto "desmercantilizador" de las políticas sociales, ya que desligan el bienestar del receptor de su capacidad de acceso a bienes y servicios producidos en el mercado, debilitando el nexo monetario entre la satisfacción de sus necesidades básicas y la venta de su fuerza de trabajo en el mercado laboral. Cuanto mayor es el nivel de desmercantilización mayor es grado en el que el bienestar individual se excluye de una esfera puramente mercantil y se garantiza sobre una base de derechos. En esta perspectiva, analizar la extensión horizontal de una política social sólo permite evaluar la amplitud del acceso a la misma, mientras es necesario discutirla también en términos de su extensión vertical, es decir, respecto al grado de protección que efectivamente brinda a cada uno de los destinatarios de la política.

Es a partir de estas consideraciones, que en este trabajo se estima la variación en términos reales del valor de las prestaciones mínimas y se comparan con la línea de pobreza argentina, denominada canasta básica total $(\mathrm{CBT})^{8}$, cuyo monto supone una aproximación, por la manera en cómo se calcula, de los bienes y servicios necesarios para satisfacer las necesidades mínimas de un individuo. Este indicador permite evaluar el objetivo de proteger a las personas mayores del riesgo de pobreza. Además se quiere evaluar el objetivo del aseguramiento frente a la pérdida de salarios al final de la vida laboral. Por esta razón se efectuará una comparación entre los haberes mínimos y los incrementos en el salario mínimo y entre los haberes medios y los ingresos medios del asalariado del sector formal, para poder estimar el grado de sustitución de salarios de las prestaciones previsionales en la parte baja y media de la distribución.

Para estimar estos indicadores, se ha debido tratar con los problemas de medición de la tasa de inflación que se han dado a partir de la intervención oficial del INDEC en 2007, y que han afectado al conjunto de las estadísticas del INDEC como bien señala Lindenboim (2011). En este trabajo se utilizará una estimación propia, denominada IPC4p, a partir de los datos publicados por 4 provincias del interior argentino, sobre la base de la metodología de Barbeito (2010), que será utilizada en alternativa a la serie de precios oficial (IPC), para los datos posteriores a 20079. Para tener una idea de la distorsión en los niveles de precios oficiales, esta estimación arroja un promedio de la tasa de inflación anual cercano al 22\% entre 2007 y 2012, contra cifras oficiales que la sitúan en torno al $9 \%$ anual. Se elige adoptar esta medida (IPC4P), a la hora de estimar el valor real de las prestaciones previsionales posterior a 2007, por ser más realística (y en línea con las estimaciones que se utilizan en la literatura). De la misma

7 Se trata de un compromiso que el gobierno argentino ha tomado en el marco de la implementación de pisos de protección social promovida por la Organización Internacional del Trabajo (OIT, 2012), para garantizar el derecho a la seguridad social sancionado por el régimen internacional de derechos humanos.

8 La CBT representa el valor monetario de la canasta de bienes y servicios utilizada como base de comparación en el cálculo de los indicadores de pobreza e indigencia en las estadísticas oficiales argentinas. Debe recordarse que la CBT se calcula a partir de la Canasta Básica Alimentar, necesaria para satisfacer las necesidades calóricas de un hombre adulto de entre 30 y 59 años (denominado adulto equivalente), a través de coeficiente de Engel, es decir, la proporción del gasto en alimentos respecto al gasto total. Posteriormente, utilizando la tabla de equivalencias del INDEC (Morales, 1988), se calcula el número de adultos equivalentes de cada hogar, según el género y la edad de cada componente.

9 Para calcular la IPC4p se toma el promedio, ponderado por la población, de la variación mensual del IPC publicada por las Direcciones Provinciales de Estadística y Censos de las provincias argentinas de Chubut, Neuquén, San Luís y Santa Fe (hasta siete provincias había dejado de publicar estos datos a lo largo de los años). Respecto al Barbeito (2010), cuya serie llegaba hasta marzo de 2010 , se extendió el período de análisis de enero de 2006 hasta agosto de 2012. La serie completa del IPC4p está contenida en Messina (2013).

GESTIÓN Y ANÁLISIS DE POLÍTICAS PÚBLICAS, Nueva Época, nº 11 enero-junio 2014 ISSN: 1989-8991 - DOI: 10.24965/gapp.v0i11.10177 
forma se revaloriza la canasta básica total (CBT), de modo que sea posible comparar la evolución de las pensiones mínimas en términos de líneas de pobreza (LP).

A continuación, se presentará una breve reflexión en torno a la trayectoria histórica del sistema de seguridad social argentino y las transformaciones que sufrió en los años 90, a causa de las reformas estructurales que la afectaron y el empeoramiento de la situación de los mercados laborales. En la sección sucesiva se presentan las principales reformas implementadas a partir de 2003 y sus efectos en términos de cobertura. Por último, se estiman los cambios observados en los niveles de las prestaciones mínimas y medias. Las dos secciones permiten evaluar las dos dimensiones, horizontal y vertical, del sistema de protección social contra los riesgos asociados a la vejez, en el componente de ingresos monetarios.

\section{LA NATURALEZA DEL SISTEMA PREVISIONAL ARGENTINO Y SUS TRANSFORMACIONES EN LA DÉCADA DE LOS 90}

El análisis que se presenta a lo largo del artículo se apoya en un marco teórico que hace referencia a la literatura sobre regímenes de bienestar, entendidos como la matriz institucional (arreglos, políticas y prácticas institucionales) conformada por los mercados, el Estado, las familias y organizaciones de la sociedad civil, cuya configuración genera efectos en términos de bienestar y de estratificación social (Gough y Wood, 2004). El análisis de los regímenes de bienestar aborda, pues, las modalidades con las que las instituciones del Estado, por medio de marcos regulatorios o de mecanismos de extracción y redistribución de recursos (que toman la forma de transferencias directas o provisión pública de bienes y servicios), intervienen sobre los resultados sociales que derivan del funcionamiento de los mercados, en particular el laboral, constituyéndose como el principal mecanismo de estratificación social en las sociedades capitalistas. A la vez, como ya se dijo en la introducción, el Estado puede reclamar para su propia responsabilidad la provisión de un conjunto de bienes, servicios, y relaciones sociales, como fundamentos materiales de los derechos sociales básicos, promoviendo una mayor o menor desmercantilización de las políticas sociales que implementa (cfr. Gamallo y Arcidiácono, 2012).

El nivel de desmercantilización inscrito en el funcionamiento de una determinada política social dependerá, en términos muy generales, del principio que regula las condiciones de acceso a la misma: necesidad, contribución y ciudadanía. A cada uno de ellos se asocia un modelo- o tipo ideal- de Estado de bienestar, según cuanto propone Esping-Andersen (1993). Al principio de necesidad, el modelo liberal, en el que el Estado asume una responsabilidad residual a favor de las personas que quedan excluidas del mercado de forma involuntaria, los "pobres merecedores". El diseño de las políticas sociales procurará entonces reducir al mínimo su efecto sobre los incentivos individuales, para no interferir en el funcionamiento de los mercados, resultando en un bajo grado de desmercantilización. En el modelo conservador-contributivo opera un mecanismo de seguro colectivo, donde la redistribución se resuelve de forma horizontal, a lo largo de categorías ocupacionales, y las condiciones de acceso pasan a depender del estatus laboral del receptor e, indirectamente, de los vínculos familiares con éste. El objetivo en este modelo es preservar el orden socioeconómico (y de género), por ende la desmercantilización es intermedia. Por último, el socialdemócrata, donde las políticas sociales son universalistas y se rigen por el principio de la titularidad de los derechos de ciudadanía, produciéndose el grado máximo de desmercantilización. Naturalmente, en el caso concreto de cada país lo que se manifiesta son configuraciones híbridas a nivel de cada sector de políticas públicas (salud, pensiones, asistencia social, etc.), tanto en términos de los principios que las regulan, como de las entidades que gestionan su funcionamiento y la erogación de bienes y servicios, así como de su financiación (cfr. Goodin y Rein, 2001).

Pese a que los regímenes de bienestar se desarrollan sobre la base de trayectorias históricas ${ }^{10}$ y tienden a mostrar cierta resiliencia frente a los cambios de corto plazo en el contexto que las rodea (Pierson, 1996), las transformaciones demográficas y de la economía capitalista han puesto en entredicho los fundamentos de los Estados de bienestar (Esping-Andersen, 2000; Taylor-Gooby, 2004). En particular, la crisis de la sociedad de pleno empleo debilitó a la principal fuente de financiamiento, y a la vez de consenso político, que alimentaba a sus instituciones, es decir las clases trabajadoras asalariadas y organizadas (Korpi, 2003). Al tiempo que el mercado laboral se fue fragmentando, excluyendo a cada vez más trabajadores del acceso a empleos con derechos sociales plenos, se vio afectado el equilibrio de los regímenes de bienestar en dirección de un mayor peso del mercado, más allá de que la estructuras y los principios rectores de las políticas sociales fueran reformados sólo parcialmente (Clayton y Pontusson, 1998).

En el caso de Argentina, el incremento de la exclusión social a lo largo de la década de los 90 fue fruto de un proceso con estas características. La expulsión del mercado formal para amplios sectores de los trabajadores significó la pérdida de titularidad de los derechos de tipo contributivo, típicos de la estructura tradicional de tipo

10 Algo que los autores anglosajones denominan dependencia del sendero (path dependence).

GESTIÓN Y ANÁLISIS DE POLÍTICAS PÚBLICAS, Nueva Época, n 11 enero-junio 2014 ISSN: 1989-8991 - DOI: 10.24965/gapp.v0i11.10177 
conservador de la Seguridad Social (Hintze, 2007), al tiempo que los procesos de individualización del ahorro, tanto en el ámbito previsional como para el seguro de salud, expandieron el peso del sector privado entre las clases medias. Por lo demás, la acción del Estado mantuvo un papel residual, por medio de programas asistenciales que estuvieron focalizados a atender a las necesidades de los sectores más vulnerables (Barrientos, 2009)11. Este proceso se inscribe en las transformaciones que experimentó el patrón de crecimiento del país en esa década, en términos de la destrucción de la sociedad asalariada preexistente (Basualdo, 2011).

Merece analizar más en detalle este proceso para tener clara las bases sobre las que actuaron los gobiernos posteriores a 2003. Debe recordarse que el sistema de Seguridad Social fue constituyéndose en Argentina, como en los países europeos de desarrollo tardío, a lo largo del siglo XX, aunque alcanzó a las clases trabajadoras sólo en la segunda posguerra, situándose el país entre los países pioneros de América Latina (cfr. Mesa-Lago, 1983). En esos años, fue construyéndose un Estado de bienestar sui generis que tomó como modelo el sistema de seguro social de la Europa continental. Si bien nunca alcanzó cobertura universal, excepto en algunos componentes, como el sistema educativo primario y el sanitario de base, permitió garantizar una cobertura muy elevada de la población, gracias al pleno empleo de los jefes varones de hogar. Se trató, por lo tanto, de un proceso que, sin lograr nunca el grado de institucionalización de los Estados de bienestar europeos, produjo una “democratización del bienestar" real (Torre y Pastoriza, 2002).

Este proceso condujo, según Filgueira (1998) a que a finales de los 70 gran parte de la población argentina estuviera cubierta por una seguridad social de tipo contributivo, aunque debe decirse que la cobertura del riesgo y el nivel de beneficios estaban fuertemente estratificados ya que el sistema estaba fragmentado en fondos destinados a cada grupo ocupacional, lo que significaba un bajo nivel de solidaridad interprofesional, hecho que terminaba privilegiando a los sectores de mayores ingresos ${ }^{12}$. Así el sistema argentino recalcaba las instituciones del sistema conservador, pero de manera imperfecta, de forma parecida a cuanto ocurrió en los países del sur de Europa, existiendo picos de generosidad muy elevada para pocos grupos privilegiados, junto a una base amplia de trabajadores formales cubierta y otros grupos que quedaron excluidos del mercado laboral formal y, por lo tanto, de la seguridad social, bajo el amparo de esquemas de tipo asistencial y clientelista (Ferrera, 1996). Sin embargo, mientras duró el régimen de pleno empleo característico del país, hasta mediados de los años 80 en términos generales, las personas excluidas del sistema contributivo constituyeron un grupo minoritario.

La década de los 90 representa, en este sentido, un periodo de ruptura de los patrones socio-económicos del país, con profundos efectos sobre el funcionamiento de la seguridad social. En primer lugar, las medidas de política económica llevadas adelante bajo la presidencia de Carlos Menem (1989-1999) tuvieron un efecto negativo sobre el mercado laboral argentino ${ }^{13}$. En esos años, se observó la reducción del número de puestos de trabajo protegidos en el sector formal, y la paralela multiplicación del desempleo y la expansión de formas no reguladas de empleo para una parte muy significativa de la población activa (Figura 1). El incremento de los procesos de exclusión social en el mercado laboral, en particular de los trabajadores no registrados, es decir que no cotizan a la Seguridad Social, provocó un desfinanciamiento del sistema, ya que significó una caída considerable de los afiliados ${ }^{14}$.

\section{FIG. 1 INDICADORES DE EXCLUSIÓN SOCIAL EN EL MERCADO LABORAL ARGENTINO}

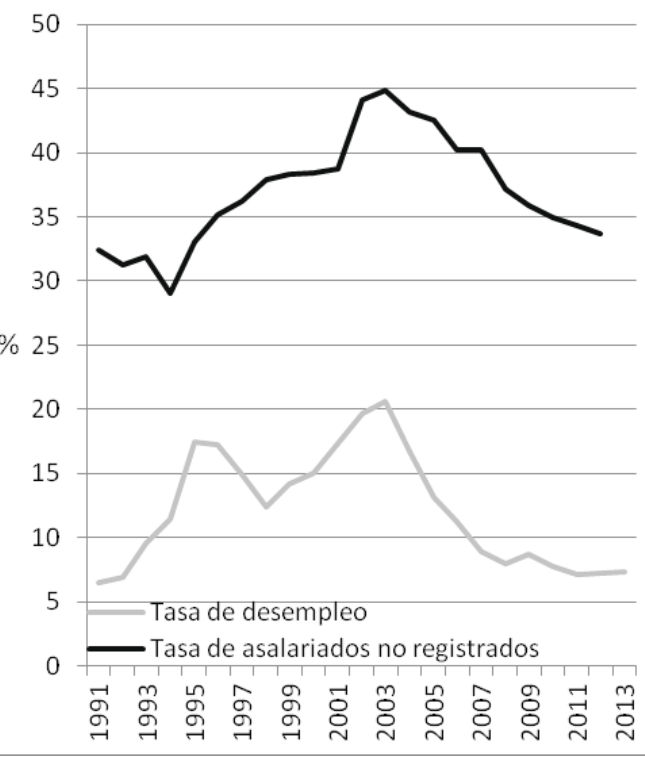

Fuente: SEDLAC (CEDLAS yBanco Mundial)

11 Por su parte, Martínez Franzoni (2008) define Argentina como uno de los regímenes de bienestar "productivistas", donde los sectores de menores ingresos acuden a los servicios públicos focalizados, mientras una proporción significativa de la población (clases medias y altas) satisface sus necesidades a través de la compra de servicios en el mercado.

12 Se trata de un fenómeno común entre los países pioneros de América Latina, donde los sistemas de seguridad social evolucionaron según un modelo de agregación de categorías ocupacionales y progresiva cobertura de riesgos, fruto de la presión y de los intereses de los grupos sociales más poderosos, que dio lugar a un modelo piramidal en el que los estratos más favorecidos gozaban de las mejores prestaciones sociales, y una base más o menos larga (según qué país) de trabajadores informales o con contribuciones insuficientes quedaban totalmente desprotegidos (Mesa-Lago, 1983).

13 Se hace referencia, en particular, a los procesos de abertura comercial indiscriminada, desregulación económica, desindustrialización y a la reducción del empleo público por la privatización de empresas estatales.

14 Los datos oficiales recogen sólo la tasa de no registro entre los asalariados, pero el porcentaje de trabajadores por cuenta propia excluidos de la seguridad social es sistemáticamente superior (cfr. Bertranou et al., 2011). La cobertura geográfica de los datos es creciente en el tiempo, al depender de la encuesta de hogares del INDEC (hasta 1991 limitada al Gran Buenos Aires, se fue ampliando hasta incluir los 31 
Las figuras permiten anticipar que el periodo posterior a la crisis de 2001-2002, objeto de la sección 3, constituye la contracara de ese proceso, ya que esta etapa ha estado marcada por una mejora importante de los indicadores laborales, gracias a una coyuntura económica muy positiva, con tasas de crecimiento medio superiores al 7\% anual entre 2003 y 2012 (INDEC). En este contexto, se ha producido una expansión del empleo registrado, con derecho a la Seguridad Social de tipo contributivo, lo que explica la recuperación de la cobertura del sistema.

En segundo lugar, el gobierno de Menem intervino en el propio funcionamiento de la Seguridad Social para hacer frente a las dificultades de financiación que el sistema arrastraba desde la década de los 80 . Estas dificultades derivaban principalmente de la grave crisis económica que afectó al país en esos años, más que de las transformaciones demográficas observadas ${ }^{15}$. Debe decirse que frente a los proyectos más liberalizadores impulsados desde los foros internacionales que propugnaban la adopción de un modelo con un pilar de capitalización individual sobre la base del ejemplo chileno (cfr. por ej. Banco Mundial, 1994), fue en ámbitos como la Seguridad Social donde con más dificultad y menor alcance el ímpetu liberalizador de administración de Menem pudo expresarse, quizás porque los sectores sindicales aliados con el oficialismo tuvieron éxito, para frenar los cambios que pudieran afectar al núcleo de sus intereses corporativos y económicos (Murillo, 2005).

De hecho, la reforma del sistema previsional argentino finalmente implementada a mediados de los años 90 se decantó por un sistema mixto que no afectaba a los derechos adquiridos por los trabajadores que lograran mantenerse en el sector regulado. Así, la sanción de la Ley $24.241^{16}$ de 1994 creó dos subsistemas dentro del Sistema Integrado de Jubilaciones y Pensiones (SIJP): un pilar público de reparto bajo la gestión de la ANSES (Administración Nacional de la Seguridad Social), en continuidad con el régimen anterior; y un sistema de capitalización individual de los aportes administrado por entidades privadas, denominadas Administradoras de Fondos de Jubilaciones y Pensiones (AFJP). La libertad de optar por uno de los dos sistemas estaba sesgada a favor del régimen de capitalización, ya que era posible pasar del sistema público al sistema privado, pero no viceversa ${ }^{17}$ (Curcio, 2011:37-38).

En lo que respecta a las pensiones erogadas, el sistema establecía una Prestación Básica Universal (PBU), a cargo del sistema público, que garantizaba una transferencia monetaria de monto mínimo similar para todos los asegurados que cumplieran con los necesarios requisitos de edad y de aportes realizados. Debe decirse que la reforma endureció estos requisitos elevándose la edad de jubilación de 60 a 65 años para los hombres y de 55 a 60 años para las mujeres, a la vez que incrementaba los años mínimos de contribución de 20 a 30. Naturalmente, esto significó excluir de la prestación a las personas que hubieran tenido una vida laboral irregular, con largos periodos de inactividad o trabajo informal, de modo que la densidad de sus contribuciones ${ }^{18}$ era insuficiente. Huelga decir que las mujeres fueron la categoría más afectada por esta medida, tanto que Massa y Fernández Pastor (2007) calculan que en 2004 cerca del $72 \%$ de los más de dos millones de personas mayores sin protección eran mujeres (en ese año, el ANSES erogaba sólo 1,65 millones de pensiones).

Regresando a la arquitectura de la reforma de 1994, la nueva ley establecía que a la PBU se sumara una prestación diferenciada según el subsistema que la persona hubiera elegido: una Prestación Adicional por Permanencia en el caso del régimen de reparto o una Jubilación Ordinaria en el caso del nuevo régimen de capitalización. Todos los trabajadores que hubieran aportado al antiguo sistema previsional recibían además una Prestación Compensatoria, en proporción a las contribuciones acumuladas en el régimen anterior (Curcio, 2011:37-38).

La institución de un sistema mixto público-privado con estas características tuvo una serie de efectos negativos ya en el corto plazo sobre la sostenibilidad y, como se anticipó, sobre la cobertura del sistema previsional argentino. Respecto a la primera cuestión, la reforma provocó un incremento del déficit fiscal del Estado, ya que el sistema público perdió los aportes de los afiliados que decidieron optar por el nuevo sistema privado, pero al mismo tiempo, el Estado mantuvo la erogación de las pensiones para las clases pasivas existentes, lo que provocó que la liquidación de los haberes fuera cubierta con el recurso a la fiscalidad general. Respecto a la financiación del sistema debe añadirse que se amplió la carga contributiva directa sobre los salarios de los trabajadores afiliados, ya que la reforma redujo los aportes patronales en casi un 60\%, con el objetivo de promover la creación de nuevos puestos de trabajo reduciendo los costos laborales para las empresas. Además, en el caso del sistema privado, los trabajadores tuvieron que pagar comisiones superiores al 30\% de sus aportes a cambio de los servicios proveídos

principales aglomerados urbanos).

15 Argentina es en efecto uno de los países donde la transición demográfica está más avanzada, pero la proporción de personas mayores de 65 años se mantiene en niveles relativamente sostenibles, aunque crecientes, pasando del 8,2\% en 1980 al 10,2\% en 2010 (INDEC, 2012).

16 Toda la legislación argentina a la que se hace referencia a lo largo del texto está disponible en la página web: http://www.infoleg.gov.ar/.

17 Además, los trabajadores que no declararon su opción fueron traspasados al sistema privado.

18 Es decir el cociente entre el tiempo cotizado efectivamente y el tiempo potencial de su vida laboral activa. 
por las AFJP para gestionar sus cuentas de capitalización, algo que en el largo plazo significaba prestaciones de menor cuantía.

Por lo que concierne la cobertura, la reforma no logró incrementar significativamente el número de los aportantes, principalmente a causa de la precarización de los ocupados y el desempleo, y en consecuencia la cobertura del sistema disminuyó a lo largo del período. Según los datos de Cetrángolo y Grushka (2004), a finales de la década más de un tercio de los mayores de 65 años estaban excluidos de cualquier tipo de cobertura previsional, contra un valor inferior al $25 \%$ en 1994 . Respecto al grado de afiliación de la población activa, a finales de la década sólo un tercio de la misma estaba cotizando al sistema, contra una cifra cercana a la mitad del total en 1994. En los hechos, la situación afectaba a los derechos previsionales futuros de buena parte de la población. Frente a esta situación, el Estado argentino se vio obligado a garantizar un nivel mínimo de ingresos para los mayores de edad, a través de programas de pensiones no contributivas asistenciales (como la pensión por vejez para mayores de 70 años sin otro ingreso). Además, el hecho de que los sistemas de capitalización garantizaran una pensión bastante inferior al último salario, llevó al Estado argentino a tener que financiar, como se dijo, la institución de una prestación mínima universal, la PBU, con el objetivo de garantizar un nivel mínimo de haberes para todos los beneficiarios del sistema contributivo.

En conclusión, la reforma de 1994 intentaba corregir el funcionamiento de un sistema de reparto que, ya en los años 80, mostraba signos de agotamiento: aun con niveles elevados de contribución, los aportes no cubrían las erogaciones, y el Estado financiaba el sistema recurriendo a otros fondos de variada naturaleza (impuestos específicos, otros fondos fiscales, etc.), manteniendo no obstante una nutrida deuda respecto a los beneficiarios, por incumplimiento de pagos (cfr. Cetrángolo y Grushka 2004). Por otra parte, el gasto en previsión social tendía a ser regresivo, ya que como se vio estaba ligado de forma directa al empleo registrado y al flujo de contribuciones aportadas a lo largo de la vida laboral. Con frecuencia, una historia laboral irregular, conformada por períodos de empleo no registrado intercalados por episodios de desempleo, impedía que muchos trabajadores pudieran reclamar una prestación al momento del retiro de la vida laboral ${ }^{19}$, mientras al mismo tiempo toda la población estaba financiando el sistema a través de sus impuestos, en particular los impuestos indirectos al consumo. Frente a estos problemas, la reforma fracasó en cuanto no logró incrementar significativamente el número de los afiliados, ni la cobertura del sistema, ni mucho menos reducir el gasto del Estado o la regresividad del sistema previsional.

\section{POLÍTICAS DE INCLUSIÓN PREVISIONAL DURANTE EL KIRCHNERISMO: EL INCREMENTO DE LA COBERTURA DEL SISTEMA DE LA SEGURIDAD SOCIAL}

La acción del gobierno argentino a partir de 2003 ha estado caracterizada por una recuperación del tradicional paradigma trabajo-céntrico como orientación básica de la política social y a la figura del trabajador asalariado como núcleo central de su estrategia de inclusión. En esta perspectiva, el empleo remunerado constituye el “eje integrador y espacio de canalización de las necesidades individuales y sociales” (Arcidiácono, 2012:26). Las situaciones de pobreza serían entonces transitorias, aunque afecten a una parte considerable de la población, y los programas sociales no contributivos representarían sólo una respuesta de corto plazo a una situación de necesidad contingente. La única solución de largo plazo a estas situaciones de vulnerabilidad pasa por el acceso de los hogares receptores al mercado de trabajo. Por otra parte, el gobierno es bien consciente de que el ritmo de generación de empleo protegido está estrechamente ligado a los niveles de actividad económica, y éstos al crecimiento de la demanda interna, en el actual patrón de acumulación. En este contexto, no es razón menor para el gobierno incrementar la capacidad de consumo de los sectores de menores ingresos por medio de una expansión de las políticas de transferencia de ingresos. La política social asistencial resultó, entonces, directamente funcional a la estrategia centrada en la inclusión social por medio del trabajo remunerado ya que, en palabras de la presidenta Fernández, medidas para "para ampliar y mejorar la cobertura social” sirven "también para incentivar la demanda agregada, el consumo y la actividad económica”"20.

Dicho esto, en el caso del sistema previsional, y en términos muy generales, el objetivo prioritario de las reformas impulsadas por el kirchnerismo en esta área ha sido incrementar la cobertura del sistema, que tanto la reforma previsional de 1994 como la situación en el mercado laboral habían reducido de forma significativa, como se vio en la sección anterior. Este objetivo ha sido perseguido a través de un compromiso fuerte del Estado, en términos de recursos invertidos, y una recuperación de su centralidad en la gestión de la previsión social. Un primer paso en la modificación del sistema mixto centrado en las AFJP, fue la eliminación de la norma que impedía el retorno al sistema público de los afiliados del sector privado (Ley 26.222 de 2007 de Libre Opción del Régimen Jubilatorio). A

19 Naturalmente todo el trabajo de cuidados, no remunerado, ejercido dentro del hogar principalmente por las mujeres no estaba reconocido por el sistema previsional.

20 En línea: http://www.presidencia.gob.ar/discursos/26491-anuncio-de-aumentos-en-asignaciones-y-medidas-para-ampliar-la-proteccion-social-palabras-de-la-presidenta-de-la-nacion (consulta: 4 de junio de 2013).

GESTIÓN Y ANÁLISIS DE POLÍTICAS PÚBLICAS, Nueva Época, n 11 enero-junio 2014 ISSN: 1989-8991 - DOI: 10.24965/gapp.v0i11.10177 
continuación, en el año 2008, con la Ley 26.425 se procedió a la renacionalización del sistema de pensiones: de esta forma se unificaron los dos sistemas preexistentes (privado de capitalización y público de reparto) en un sistema público único, denominado Sistema Integrado Previsional Argentino (SIPA)21, que pasó a recibir todos los aportes, absorbiendo los fondos de pensión administrados por las AFJP. Los activos de las AFJP fueron traspasados por Decreto 897 de 2007 al Ilamado Fondo de Garantía de Sustentabilidad (FGS) del régimen público, manejado por el ANSES. El efecto de estas medidas fueron por lo tanto el de incrementar notablemente la financiación del sistema previsional público, tanto por el crecimiento del número de afiliados, gracias a la recuperación del mercado laboral, como por la adquisición de reservas que habrían de garantizar la sostenibilidad futura de las pensiones.

Cabe recordar que por largo tiempo una parte considerable de los recursos del sistema contributivo no provino de las cotizaciones de los afiliados sino de la propia fiscalidad general ${ }^{22}$. En este sentido, la ampliación de la cobertura a favor de los no aportantes ha producido una mayor progresividad del régimen previsional. Sobre la base de estos hechos también se entiende que el sistema de la Seguridad Social argentino ya no cumple sólo con la función clásica de seguro colectivo obligatorio, es decir aquel en el que el flujo de cotizaciones (la prima) garantiza el derecho futuro a recibir una pensión de vejez una vez alcanzada la edad legal (y el número mínimo de años de cotización). Más bien, y siguiendo a Barr (2003), no sólo funciona como una "hucha" ('piggy bank') colectiva, regulada por el Estado, para que las personas redistribuyan sus ingresos a lo largo de su ciclo vital, trasfiriendo recursos desde su vida laboral activa al periodo de la jubilación²3, sino que también cumple la función de "Robin Hood", ya que persigue objetivos como la provisión de sustento para los más pobres, la redistribución del ingreso y la reducción de la exclusión social, en el caso específico, a favor de las personas mayores.

Con una breve digresión, merece decirse que en el caso argentino existen seguros colectivos de salud de tipo contributivo, denominados "obras sociales", que cumplen la función de cubrir los tratamientos sanitarios de los afiliados, y constituyen uno de los componentes fundamentales de la seguridad social, estrechamente ligados al sistema previsional, ya que ambos son financiados por aportes de trabajadores y empleadores. Dentro del sistema de las obras sociales, las personas mayores que reciben una prestación previsional contributiva pasan a recibir la cobertura sanitaria del Instituto Nacional de Servicios Sociales para Jubilados y Pensionados - INSSJP (denominada PAMI - Programa De Atención Médica Integral). Por esta razón existe una correlación directa entre el incremento de la cobertura previsional y la ampliación del seguro social de salud para los trabajadores retirados. En otras palabras, las políticas de inclusión previsional no sólo fueron en la dirección de garantizar el acceso al derecho a una protección contra la pérdida de ingresos asociada al fin de la vida laboral activa, sino que también contribuyeron a garantizar el derecho a la salud de las personas mayores.

Dicho esto, a continuación, se discutirán con más detalle otras medidas que afectaron a la cobertura del sistema previsional bajo los gobiernos de Néstor Kirchner y Cristina Fernández, ampliando la cobertura del sistema previsional de forma notable. En particular, deben mencionarse la “moratoria previsional” (Decreto 1454 de 2005 ), denominada oficialmente "Plan de Inclusión Previsional", para permitir que las personas en edad de jubilación, con un número insuficiente de años de cotización, pudieran ingresar al sistema de reparto público24; la prejubilación de los trabajadores en situación de desempleo (Ley 25.994 de 2004); el régimen de cotización simplificado para trabajadores autónomos (monotributo), en particular aquellos en situación de vulnerabilidad (monotributo social); la promoción de la regularización del trabajo doméstico a raíz de la institución de un régimen tributario especial para esa categoría laboral (Ley 26.063 de 2005) y la nueva legislación laboral que regula el sector (Ley 26.921 de 2013).

21 Debe recordarse que el SIPA incluye todos los sistemas de la seguridad social nacional: régimen general, algunos regímenes especiales (poder judicial de la nación, personal docente o universitario, etc.), además de ex-cajas provinciales transferidas a la nación. No incluye las cajas provinciales, cajas previsionales de algunas profesiones, o el régimen especial de las fuerzas armadas y de las fuerzas de seguridad. (OSS, 2011). Los afiliados a estos regímenes no están incluidos en la figura 2, pero habrían alcanzado alrededor de 1.5 millones de personas en junio de 2012 (SSS, 2012).

22 Entre el año 2000 y el 2009, el sistema previsional nacional fue financiado, en promedio, sólo en un 46,7\% por aportes de los trabajadores activos mientras el resto estuvo financiado por otros ingresos dentro de la fiscalidad general, donde tuvo lugar predominante el IVA, impuesto esencialmente regresivo, (GESS, 2010: 11), aunque la recuperación del mercado laboral ha hecho que la financiación vía contribuciones cubra actualmente una parte mayoritaria del gasto (MECON, 2012).

23 Según esta perspectiva, el Estado trataría, con cierto grado de paternalismo, a los ahorros previsionales como "bienes meritorios" ('merit goods'), es decir bienes que la "miopía” individual (el mayor peso del presente respecto al futuro en las preferencias de consumo de las personas) hacen acumular en manera insuficiente, y por lo tanto requieren de alguna regulación pública. Según otro punto de vista (Castel, 1997: 311), el seguro social fue constituido como una "cuasi propiedad" que extendía a los trabajadores asalariados la principal ventaja de la que había tradicionalmente disfrutado los poseedores de alguna propiedad privada, es decir, su uso como cobertura frente a riesgos vitales. De esta forma, los asalariados superaban su condición de desamparados y adquirían el respaldo de toda la sociedad cada vez que sus ingresos se veían interrumpidos por alguna de las causas establecidas.

24 Paradójicamente, los participantes de la moratoria subscribieron planes de amortización de las contribuciones faltantes, hasta un máximo de 60 cuotas, que son descontadas de las prestaciones que reciben mensualmente, convirtiéndose en cierto sentido en deudores del sistema, a cambio de ver cumplido su derecho a una protección de los riesgos asociados a la vejez.

GESTIÓN Y ANÁLISIS DE POLÍTICAS PÚBLICAS, Nueva Época, n 11 enero-junio 2014 ISSN: 1989-8991 - DOI: 10.24965/gapp.v0i11.10177 
FIG. 2. AFILIADOS AL RÉGIMEN PREVISIONAL NACIONAL Y TITULARES DE UNA PRESTACIÓN

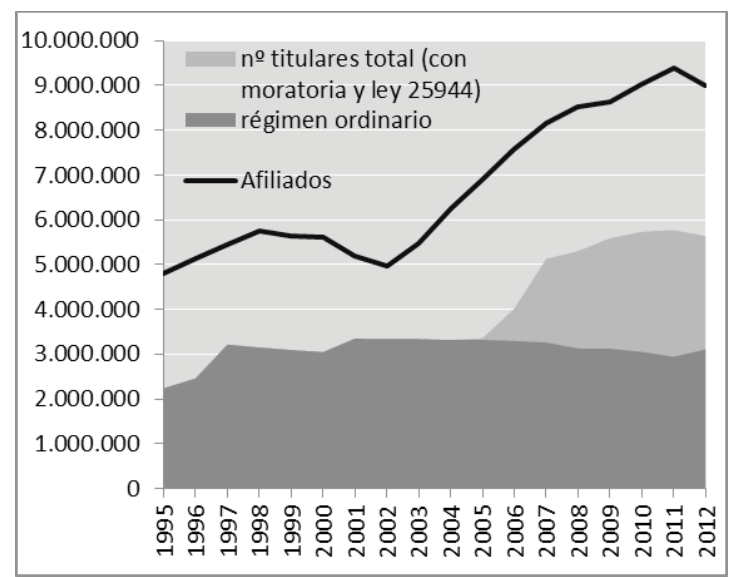

Fuente: SSS(2012) y MECON (2013)

Gracias a la moratoria, el número de titulares de una prestación previsional registró una subida considerable, como se observa en la Figura 2. De hecho, en ausencia de la moratoria no se habría registrado ningún incremento en términos absolutos de la cobertura previsional entre la población pasiva. Más de 2 millones y medio de personas adhirieron a la moratoria (el $45 \%$ de los afiliados en 2012), mientras el régimen ordinario de jubilación mantenía alrededor de 3 millones de titulares a lo largo de todo el periodo. De esta forma, y como resultado de esta medida, la cobertura del conjunto de la previsión social argentina alcanzó a finales de 2011 una cobertura cercana al 95\% de la población mayor de 65 años ${ }^{25}$ (cfr. SSS, 2012). Debe añadirse, que cerca del 72\% de los beneficiados por la moratoria previsional fueron mujeres, de ahí que recibiera en los medios el nombre despectivo de "jubilación de la ama de casa". Dejando de lado estas expresiones, es indudable que, desde una perspectiva de género, la medida supuso un avance en el reconocimiento de los derechos de las mujeres.

Volviendo al balance de una década, por lo que concierne el número de trabajadores en activo afiliados, el incremento fue de más de 4,4 millones de unidades en comparación con el valor mínimo de la serie registrado en diciembre de 2002 y de más de 3,6 millones respecto al máximo registrado durante la convertibilidad (diciembre de 1998). Está claro que la coyuntura económica muy positiva de estos años tuvo un papel fundamental para alcanzar estas cifras. El nivel de cobertura sobre la población económicamente activa fue del $54 \%$ aproximadamente en diciembre de 2011, gracias a 3 millones de nuevos aportantes en la categoría de los trabajadores dependientes, dato que confirma el crecimiento significativo del empleo registrado (pero también los desafíos de la persistencia de un sector informal muy amplio, cfr. Bertranou et al., 2011). Así las cosas, las medidas de simplificación y regularización del trabajo no formal tuvieron no obstante un impacto positivo, ya que se pudo observar un incremento de más de 900 mil unidades en el caso de los trabajadores autónomos incluidos en el régimen del monotributo, de los cuales cerca de la mitad inscritos al régimen del monotributo social (datos MDS, 2011); además, cerca de 250 mil personas ingresaron al régimen especial para trabajadoras domésticas (SSS, 2012).

\section{LA ADECUACIÓN DE LAS PRESTACIONES A LOS OBJETIVOS DEL SISTEMA PREVISIONAL}

Si en términos de la primera dimensión de la desmercantilización, es decir la extensión horizontal del sistema, pudo verse una mejora significativa de la cobertura a lo largo de la década, los resultados son más difíciles de evaluar en relación con los niveles de las prestaciones erogadas, es decir la extensión vertical de la previsión social. En términos nominales, los haberes mínimos fueron periódicamente incrementados, después de haber permanecido invariados en un valor de 150 pesos argentinos en los años entre 1995 y 2001 (cfr. Tabla 1) ${ }^{26}$. Si entre 2003 y 2007 los aumentos fueron fijados de forma discrecional, a partir de la sanción de la Ley 26417 de movilidad previsional (octubre de 2008) se fijaron las reglas de incremento anual de los haberes, con el objetivo de proteger el poder adquisitivo de los afiliados a lo largo del tiempo ${ }^{27}$. Pese a ello, los datos que se muestran en la Figura 3 (en pesos

25 Al tiempo que la moratoria previsional progresaba, se redujo el número de titulares de pensiones no contributivas por vejez, para mayores de 70 años sin recursos quedando en junio de 2012 sólo 38.432 titulares (eran 92.517 en 2006). Al contrario, la pensión no contributiva por invalidez creció de manera exponencial de alrededor de 80 mil titulares en 2003 a 791.150 a mediados de 2012. No existen datos sobre cuántos de ellos serían mayores de 65 años, pero sí se sabe que el valor medio de la prestación es inferior a la que garantiza el sistema previsional contributivo (SSS, 2012).

26 Además, fueron otorgados aumentos de suma fija y se fijaron algunos subsidios específicos que favorecieron a los afiliados del PAMI (OSS, 2012: 60-1).

27 Esta ley establece que los haberes se incrementen en marzo y septiembre de cada año en un porcentaje que se calcula sobre la base del promedio de los salarios reales de los diez años anteriores y de la recaudación del ANSES, garantizando un mayor grado de protección del valor real de las prestaciones.

GESTIÓN Y ANÁLISIS DE POLÍTICAS PÚBLICAS, Nueva Época, n 11 enero-junio 2014 ISSN: 1989-8991 - DOI: 10.24965/gapp.v0i11.10177 
constantes de 2001) ponen en cuestión el alcance de los incrementos otorgados, si se estima su variación en términos reales a partir de 2007 con el IPC4P propuesto en este trabajo.

Así es evidente que la caída en términos reales de las prestaciones en correspondencia a la devaluación de 2002, no ha sido todavía recuperada del todo, por lo menos en el caso del valor promedio de las pensiones. En relación a esta cuestión, sin embargo, debe precisarse que debe tenerse en cuenta un efecto composición a la hora de comparar los dos periodos. Este deriva de las diversas características de los afiliados en el sistema en los dos momentos. En el caso de los años 90, mientras que los parámetros de cálculo de los haberes no eran modificados (como muestra la evolución del mínimo), los haberes medios aumentaron porque, en mayor medida, accedieron a una prestación aquellas personas que tuvieran vidas laborales estables y salarios más elevados. El efecto contrario es visible en la última década. A causa de la moratoria, la gran mayoría de los nuevos receptores que ingresaron al sistema recibieron la prestación mínima, tanto que en 2012 más de dos tercios de los beneficiarios recibían ese monto (SSS, 2012).

Dicho esto, a partir de 2007, es visible el efecto negativo del crecimiento de los precios al consumo sobre el poder adquisitivo de las prestaciones, en particular sobre el haber mínimo (que no está afectado por el citado efecto de composición). De acuerdo a las estimaciones efectuadas para este artículo, la inflación al consumo acumulada entre 2007 y 2012 se habría situado en un valor cercano al $230 \%{ }^{28}$. Está claro que un incremento de los precios de esta amplitud licuó una parte importante de los incrementos nominales que se dieron en este periodo. Aunque en los últimos tres años parece haber funcionado el mecanismo de actualización incluido en la ley de movilidad, en 2013el nivel de los haberes mínimos se situaría, en términos reales, ligeramente por debajo del nivel observado en 2006. Por último, puede notarse que en todo caso se está hablando de niveles que en el periodo 2007-2013 estarían un 23\% por encima del promedio observado entre 1995 y 2001 para los haberes mínimos.

FIG. 3. HABERES MÍNIMOS Y MEDIOS DEL RÉGIMEN PÚBLICO (PESOS CONSTANTES-DIC 2001)

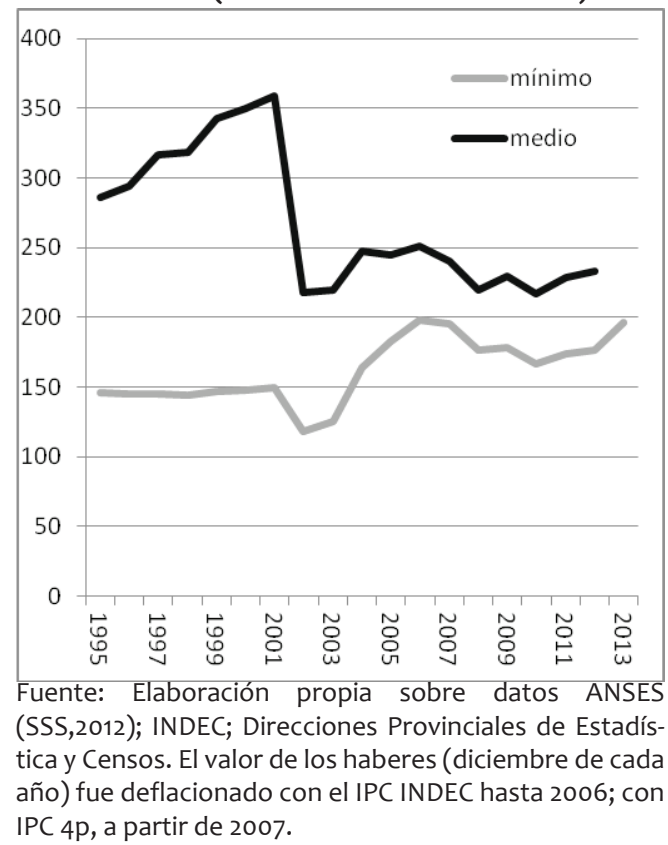

TABLA 1. MONTO DE LAS PRESTACIONES DE LA PREVISIÓN SOCIAL - COMPARACIÓN CON INDICADORES SOCIALES SELECCIONADOS

\begin{tabular}{|c|c|c|c|c|c|}
\hline & \multicolumn{3}{|c|}{ HABER MíNIMO } & \multicolumn{2}{|c|}{ HABER MEDIO } \\
\hline & Pesos corrientes & $\%$ CBT INDEC & $\% S M V M$ & Pesos corrientes & Salarios medios* \\
\hline 2001 & 150 & & $75 \%$ & 358,97 & \\
\hline 2002 & 200 & & $100 \%$ & 369,08 & \\
\hline 2003 & 220 & $95 \%$ & $73 \%$ & 387,01 & \\
\hline 2004 & 308 & $129 \%$ & $68 \%$ & 463,59 & $48 \%$ \\
\hline 2005 & 390 & $145 \%$ & $62 \%$ & 522,38 & $44 \%$ \\
\hline 2006 & 470 & $161 \%$ & $59 \%$ & 595,31 & $42 \%$ \\
\hline 2007 & 596,2 & $167 \%$ & $61 \%$ & 733,71 & $41 \%$ \\
\hline 2008 & 690 & $159 \%$ & $56 \%$ & 860,37 & $39 \%$ \\
\hline 2009 & 827,23 & $165 \%$ & $57 \%$ & $1.064,38$ & $41 \%$ \\
\hline 2010 & $1.046,43$ & $165 \%$ & $60 \%$ & $1.359,27$ & $41 \%$ \\
\hline 2011 & $1.434,29$ & $183 \%$ & $62 \%$ & $1.883,52$ & $43 \%$ \\
\hline 2012 & $1.879,67$ & $196 \%$ & $70 \%$ & 2480,20 & $48 \%$ \\
\hline 2013 & 2476,98 & $211 \%$ & $75 \%$ & & \\
\hline
\end{tabular}
HABER MÍNIMO/MEDIO \begin{tabular}{l}
$41,79 \%$ \\
$54,19 \%$ \\
$56,85 \%$ \\
$66,44 \%$ \\
$74,66 \%$ \\
$78,95 \%$ \\
$81,26 \%$ \\
$80,20 \%$ \\
$77,72 \%$ \\
$76,98 \%$ \\
$76,15 \%$ \\
$75,79 \%$ \\
\hline
\end{tabular}

*Ingreso de la ocupación principal de los asalariados registrados (Promedio) - $4^{\circ} \mathrm{T}$ cada año.

Fuente: elaboración propia. Haberes: Valores de diciembre de cada año, SSS (2012); CBT (INDEC) actualizados con IPC4p desde 2007; SMVM y Salarios medios (MTEySS).

28 Sobre la base del IPC (INDEC) la variación acumulada en el mismo periodo habría sido de sólo el $68 \%$. 
El análisis de la evolución del poder adquisitivo de las prestaciones no es suficiente para evaluar si su nivel es adecuado para cumplir los dos objetivos mencionados con anterioridad: por un lado, servir de seguro frente a la pérdida de salarios al término de la vida laboral activa; por el otro, garantizarles, por lo menos, un nivel suficiente de ingresos para adquirir una canasta de bienes y servicios básica. En relación al segundo objetivo, aquí se propone comparar el valor de los haberes mínimos con la CBT, que fija la línea de pobreza en Argentina. Respecto al primer objetivo, y para aproximar la tasa de sustitución de los salarios que asegurarían las prestaciones, se compararán los haberes mínimos con el salario mínimo regulado por ley (el Salario Mínimo, Vital y Móvil - SMVM) y los haberes medios con los salarios medios de los asalariados registrados, es decir aquellos que cotizan a la seguridad social, ya que se trata de los datos disponibles más completos proporcionados por el Ministerio de Empleo, Trabajo y Seguridad Social (MTEySS).

El resultado de este ejercicio puede verse en la Tabla 1. Los datos muestran que las nuevas medidas parecen haber establecido un piso de protección básica, ya que los receptores de una prestación mínima cubren, al final del periodo considerado, dos veces el monto de la CBT, más del doble respecto a los niveles de 2003. Respecto a la relación entre haberes y salarios, se evidencia una evolución positiva, en particular a partir de 2009, cuando fue aplicada la ley de movilidad previsional. Si bien, cuando actuaba discrecionalmente, el gobierno favoreció la recuperación de los haberes mínimos, reduciendo la brecha entre éstos y los haberes medios, más allá del mencionado efecto de la moratoria, cuando entra en vigor la ley de movilidad la relación entre ambos se mantiene relativamente estable ${ }^{29}$.

\section{CONCLUSIONES: LOS DESAFÍOS PENDIENTES}

Las secciones 3 y 4 de este artículo han desarrollado un análisis de las medidas adoptadas por los gobiernos kirchneristas para incluir a sectores previamente excluidos del sistema contributivo de pensiones. Entre las medidas examinadas, pueden destacarse la moratoria, y los efectos que tuvo en términos de ampliación de la cobertura para las personas mayores de 65 años, poniendo solución a la situación heredada de la década anterior (sección 2) y exacerbada por la crisis de 2001-2002). De hecho, los datos apuntan a que en la etapa posterior a 2003 se privilegió la extensión de la cobertura del sistema previsional a favor de las personas mayores de 65 años, respecto al gasto que habría supuesto incrementar de forma significativa el valor real de las prestaciones. Así las cosas, las prestaciones recibidas por los millones de nuevos receptores que ingresaron gracias a la moratoria se situaron en un nivel promedio cercano al mínimo. En todo caso, la ley de movilidad previsional de finales de 2008, que vincula el incremento de las prestaciones a un índice combinado de los salarios reales y los recursos de la ANSES, ha permitido una mejora significativa en este aspecto, pese al incremento considerable de los precios al consumo a partir de 2007. En particular, parece cumplirse el objetivo de garantizar a toda persona mayor unos niveles de ingreso suficientes para mantenerla fuera de la pobreza, ya que los haberes mínimos han pasado a cubrir dos veces la línea de pobreza (medida con la CBT). Menos éxito habría tenido el objetivo de garantizar una tasa de sustitución de las prestaciones coherente con los niveles salariales predominantes en el mercado laboral, aunque también en este caso la ley de 2008 tuvo un efecto positivo.

Respecto a la sostenibilidad futura del sistema, y a las posibilidades de seguir progresando en la dirección de una mayor cobertura y adecuación de las pensiones, las incógnitas son numerosas y no pueden ser tratadas aquí en detalle, dados los límites de espacio. En todo caso, es posible señalar algunos puntos clave que considerar. En primer lugar, debe recordarse que la financiación del sistema mejoró gracias al incremento de afiliados provocado por la mejora del mercado laboral, y a los efectos de las políticas de regularización e inspección laboral ${ }^{30}$. Pese a ello, como se vio, una parte importante de la financiación todavía proviene de la tributación general. Además debe recordarse el persistente problema de la informalidad laboral: además de constituir una pérdida de financiación presente también pone en riesgo las pensiones futuras de una parte considerable de los argentinos. A este respecto, hay que subrayar que la moratoria de 2005 no constituyó una reforma estructural que modificara la arquitectura contributiva del sistema, sino un parche a una situación de emergencia. Esto se explica ya que se utilizó una norma anterior, destinada a la regularización de la deuda previsional de los trabajadores autónomos (Ley 24.476 de 1995), para que muchas personas con vida laboral irregular (o, directamente, no remunerada) pudieran cubrir las cotizaciones faltantes en el periodo que llega hasta 1994, y así recibir una prestación. Esto indica que el efecto de la moratoria es sólo temporal, y que un problema de cobertura podría presentarse en un futuro próximo, a

29 La ley de movilidad también afectó al valor de las pensiones asistenciales, vinculándolas al haber mínimo previsional aunque por debajo del mismo. En particular, el valor promedio de las pensiones no contributivas de carácter asistencial se mantuvo cerca al $80 \%$ de la prestación contributiva mínima. Así las cosas, el paso progresivo al sistema contributivo para los receptores de una pensión no contributiva por vejez supuso una mejora considerable en sus condiciones.

30 Además la ley de movilidad también incrementa anualmente la base imponible de las alícuotas previsionales, al incrementar el máximo del salario gravado por la contribución.

GESTIÓN Y ANÁLISIS DE POLÍTICAS PÚBLICAS, Nueva Época, n 11 enero-junio 2014 ISSN: 1989-8991 - DOI: 10.24965/gapp.v0i11.10177 
menos que se actualizara la norma, o se modificaran estructuralmente las reglas de acceso y el funcionamiento del mercado laboral argentino.

A partir de estas consideraciones puede decirse que, pese a los avances observados, las nuevas medidas de política social no han modificado de forma significativa ni la estructura del gasto social ni las modalidades de financiación del mismo (cfr. Isuani, 2010). Por ejemplo, el régimen general de financiación a través de los aportes de trabajadores y empleadores no ha sido modificado a lo largo de la década, aunque ha habido programas de reducción temporal, en respuesta a la crisis económica internacional posterior a 2008 (OSS, 2012) 31. En este aspecto, la reforma de 1994 no ha sido revertida. El contexto económico favorable de la década ha permitido financiar, de la manera que se dijo, el sostenido crecimiento del gasto público destinado al régimen previsional. Este alcanzó un $7,7 \%$ del PIB en 2013, frente a un 4,35\% en 2003. Debe decirse que el gasto medio de la primera etapa del kirchnerismo entre 2003 y 2007 (4.3\%) fue inferior al promedio entre 1995-2001 (5,31\%), entre otras cosas, por la caída en términos reales de las prestaciones. El promedio entre 2008 y 2013 fue netamente superior (6,8\%), aunque en un contexto interno y externo de progresivo desmoronamiento del patrón de crecimiento característico de los primeros años posteriores a la crisis. Además de una reducción del ritmo de crecimiento y creación de empleo registrado, las cuentas públicas entraron en déficit, de forma continuada, a partir de 2009.

Así las cosas, si por un lado no pueden negarse los méritos de las políticas adoptadas a partir de 2003 , mantenidas incluso en el actual contexto de crisis internacional, tampoco pueden negarse los desafíos que amenazan el proceso de inclusión previsional iniciado hace ya una década. Dejando de lado problemas de largo plazo de tipo demográfico, al centro de la cuestión está el hecho que un sistema de seguridad social estructurado según principios contributivos siempre estará expuesto a los vaivenes del mercado laboral. En ese contexto, la relajación de las reglas de acceso al régimen contributivo ha sido una medida importante pero transitoria que no ha modificado los condicionantes estructurales del sistema previsional argentino, al no ofrecer soluciones de largo plazo a los grupos sociales excluidos de un empleo formal. Pese a todas las medidas implementadas, una cuota muy significativa de los trabajadores activos permanece atrapada en la informalidad. Este factor constituye un obstáculo formidable para construir desde el consenso social un sistema de políticas sociales más universales.

\section{BIBLIOGRAFÍA}

Arcidiácono, P. (2012), La política del “mientras tanto" - Programas sociales después de la crisis 2001-2002. Buenos Aires: Editorial Biblos.

Basualdo, E. (2011), Sistema político y modelo de acumulación - tres ensayos sobre la Argentina actual. Buenos Aires: Atuel.

Barbeito, Alberto C. 2010. "Re-construcción de índices de precios al consumidor y su incidencia en la medición de algunas variables monetarias reales.”, Análisis de Coyuntura, 23. Buenos Aires: CIEPP.

Barr, N. (2003), The welfare state as piggy bank: information, risk, uncertainty, and the role of the state. Oxford: Oxford University Press.

Barrientos, Armando. 2009. "Labour markets and the (hyphenated) welfare regime in Latin America”, Latin American Economy and Society, 38: 87-108.

Banco Mundial (1994), Envejecimiento sin crisis: políticas para la protección de los ancianos y la promoción del crecimiento. Washington D.C.: Oxford University Press.

Bertranou, F., Cetrángolo, O., Grushka, C. y Casanova, L. (2011), Encrucijadas en la seguridad social argentina: reformas, cobertura y desafíos para el sistema de pensiones. Buenos Aires: CEPAL y OIT.

Castel, R. (1997), Las metamorfosis de la cuestión social: una crónica del salariado. Buenos Aires, Paidós.

Cetrángolo, O. y Grushka, C. (2004), "Sistema previsional argentino: crisis, reforma y crisis de la reforma.”, Financiamiento del desarrollo, 151. Santiago de Chile: CEPAL

Clayton, R. y Pontusson J. (1998), “Welfare-State Retrenchment Revisited: Entitlement Cuts, Public Sector Restructuring, and Inegalitarian Trends in Advanced Capitalist Societies”, World Politics, 51: 67-98.

Curcio, J. (2011). “Descripción del Sistema de Seguridad Social: componentes al cabo de la década del ‘90 y de la

31 Así, al trabajador dependiente se le descuenta el 17\% de su base salarial imponible (de los cuales el 11\% va al SIPA, el 3\% a su Obra Social y el 3\% al INNSJP). El régimen general para los empleadores prevé un aporte del 23\%, que se destina al SIPA (10, 17\%), al sistema de asignaciones familiares (4,44\%), al Fondo Nacional de Empleo (0,89\%), al INSSJP (1,5\%) y a la Obra Social del trabajador (6\%). 
primera década del siglo XXI", en C. Danani y S. Hintze, Protecciones y desprotecciones: la seguridad social en la Argentina 1990-2010. Buenos Aires: UNGS.

Esping-Andersen, G. (2000), Fundamentos sociales de las economías postindustriales. Barcelona, Ariel

--- (1993), Los tres mundos del Estado del Bienestar. Valencia: Alfons el Magnanim.

Ferrera, M. (1996), “The 'Southern Model' of Welfare in Social Europe”, Journal of European Social Policy, 6 (1): 17-37.

Filgueira, F. (1998). Tipos de Welfare y Reformas Sociales en América Latina - Eficiencia, residualismo y ciudadania estratificada. Chicago: LASA.

Gamallo, G. y Arcidiácono, P. (2012). "Políticas sociales y derechos. Acerca de la producción y reproducción de las marginaciones sociales”, en L. Pautassi y G. Gamallo, dirs., ¿Más derechos, menos marginaciones? Políticas sociales y bienestar en la Argentina. Buenos Aires: Editorial Biblos.

GESS - Gerencia de Estudios de la Seguridad Social (2010), Análisis de la cobertura previsional del SIPA: Protección, Inclusión e Igualdad. Buenos Aires: ANSES.

Goodin, R.. y Rein, M. (2001), "Regimes on Pillars: Alternative Welfare state logics and dynamics", Public Administration, 79: 769-801.

Gough, I. y Wood, G. (2004), Insecurity and Welfare Regimes in Asia, Africa and Latin America. New York: Cambridge University Press.

Hintze, S. (2007), Políticas sociales argentinas en el cambio de siglo: conjeturas sobre lo posible. Buenos Aires: Espacio.

INDEC - Instituto Nacional de Estadística y Censo. En línea: <www.indec.gov.ar> (consulta: 25 enero 2014).

--- (2012), Censo Nacional de Población, Hogares y Viviendas 2010 - Tomo 1. Buenos Aires: INDEC.

Isuani, E. (2010), "The Argentine Welfare State: Enduring and Resisting Change", International Journal of Social Welfare, 19: 104-114.

Korpi, W. (2003), "Welfare-State Regress in Western Europe: Politics, Institutions, Globalization, and Europeanization", Annual Review of Sociology, 29: 589-609.

Lindenboim, J. (2011), “Las estadísticas oficiales en Argentina ¿Herramientas u obstáculos para las ciencias sociales ?”, Trabajo y Sociedad 16: 19-38.

Martínez Franzoni, J. (2008), "Welfare Regimes in Latin America: Capturing Constellations of Markets, Families, and Policies", Latin American Politics and Society, 50: 67-100.

Massa, S. y Fernandez Pastor, M. (2007), De la exclusión a la inclusión social, reforma de las reformas de la Seguridad Social en la República Argentina. Buenos Aires: Editorial Prometeo.

MECON - Ministerio de Economía (2013), Ejecución físico financiera - Presupuesto de la Administración Nacional - Acumulado $4^{\circ}$ Trimestre 2012. En línea: <http://www.mecon.gov.ar/hacienda/cgn/cuenta/2012/tomoii/jur75. htm> (consulta 24 enero 2014).

Mesa-Lago, C. (1983), "Social Security and Extreme Poverty in Latin America", Journal of Development Economics, 12: 83-110.

Messina, Giuseppe M. (2013). Desarrollo con inclusión social: coyunturas económicas y políticas públicas. El caso de Argentina en la post-convertibilidad. Tesis Doctoral. Madrid: Universidad Complutense.

MDS - Ministerio de Desarrollo Social (2011)," Más de 457 mil personas se inscribieron en el Monotributo Social”.

En línea: <http://www.desarrollosocial.gov.ar/Noticia/457milinscriptos/795> (consulta: 19 de diciembre de 2012).

Morales, E. (1988), Canasta básica de alimentos - Gran Buenos Aires, Documento de trabajo, nº 3. Buenos Aires: INDEC/IPA.

MTEySS - Ministerio de Trabajo, Empleo y Seguridad Social, Boletín de Estadísticas Laborales. En línea: <http:// www.trabajo.gov.ar/left/estadisticas/bel/> (consulta 27 enero 2013).

Murillo, M. V. (2005), Sindicalismo, coaliciones partidarias y reformas de mercado en América Latina. Madrid: Siglo $\mathrm{XXI}$. 
OIT - Organización Internacional del Trabajo (2012). Pisos de protección social para la justicia social y una globalización equitativa. Informe IV, $101^{\text {a }}$ Conferencia Internacional del Trabajo, Ginebra.

OSS - Observatorio de la Seguridad Social (2012). La inclusión social como transformación: políticas públicas para todos. Buenos Aires: ANSES.

--- (2011), Marco conceptual del sistema de estadísticas e indicadores del sistema integrado previsional argentino. Buenos Aires: ANSES.

Pierson, P. (1996), “The New Politics of the Welfare State”, World Politics, 48: 143-179.

SSS - Secretaría de Seguridad Social (2012), Boletín Estadístico de la Seguridad Social. Buenos Aires: Ministerio de Trabajo, Empleo y Seguridad Social.

Rofman, R., Apella, I. y Vezza, E. (2013), Más allá de las Pensiones Contributivas. Catorce experiencias en América Latina. Buenos Aires: Banco Mundial.

SEDLAC Socio-Economic Database for Latin America and the Caribbean (CEDLAS y Banco Mundial): En línea: <http://sedlac.econo.unlp.edu.ar/esp/estadisticas.php> (consulta: 22 de enero de 2014).

Taylor-Gooby, P. (2004), New Risks, New Welfare: the Transformation of the European Welfare States. New York: Oxford University Press.

Torre, J. C. y Pastoriza, A. (2002), "La democratización del bienestar", en J. C. Torre, dir., Los años peronistas (19431955). Buenos Aires: Sudamericana. 